\title{
Leptons, Quarks, and Their Antiparticles: A Phase-Space View
}

\author{
Piotr Żenczykowski
}

Received: 17 March 2010 / Accepted: 17 June 2010 / Published online: 30 June 2010

(C) The Author(s) 2010. This article is published with open access at Springerlink.com

\begin{abstract}
Recently, a correspondence has been shown to exist between the structure of a single Standard Model generation of elementary particles and the properties of the Clifford algebra of nonrelativistic phase space. Here, this correspondence is spelled out in terms of phase-space variables. Thus, a phase-space interpretation of the connections between leptons, quarks and their antiparticles is proposed, in particular providing a timeless alternative to the standard Stückelberg-Feynman interpretation. The issue of the additivity of canonical momenta is raised and argued to be intimately related to the unobservability of free quarks and the emergence of mesons and baryons.
\end{abstract}

Keywords Quantum geometry $\cdot$ Phase space $\cdot$ Clifford algebra $\cdot$ Structure of Standard Model generation

\section{Introduction}

In the standard formalism used for the description of elementary particles, transformations between observed particles are given within the context of various symmetry groups, such as $S U(2), S U(3)$, etc. In particular, transformations between particles and antiparticles are effected via complex conjugation, with antiparticles belonging to complex-conjugate representations of the symmetry groups in question. Complex conjugation is also needed when discussing time reversal, e.g. in the time-dependent Schrödinger equation. Thus, the existence of particles and antiparticles appears to be closely related to the existence of the macroscopic concept of external time. And indeed, Stückelberg and Feynman interpreted antiparticles as 'particles moving backward in time'.

In quantum gravity, however, the concept of external time is - as argued by many (e.g. $[6,8,10])$ - presumably the first notion to be discarded. In fact, 'time' is never measured directly, we always measure positions, be it positions of clock hands, or positions of other

P. Żenczykowski (凶)

Division of Theoretical Physics, Institute of Nuclear Physics, Polish Academy of Sciences,

Radzikowskiego 152, 31-342 Kraków, Poland

e-mail: piotr.zenczykowski@ifj.edu.pl 
groups of particles. Ultimately, we deal with relative positions only. Consequently, the 'flow of time' may be replaced by the observed regular changes in the correlations between positions of (groups of) particles [1] (regardless of which of our senses they affect). This is what astronomers actually do: they define time through changes in the positions of astronomical objects [3]. Obviously, at the deep level and irrespectively of this procedure, there should exist something that time originates from. If it is not a classical time, it might be its quantized version, a quantized version of changes in objects' relative positions, or something much more involved.

Now, when the external time of the Schrödinger equation vanishes from the formalism (in some approximation being presumably superseded by such changes in particles' positions), the rationale for complex numbers in quantum gravity seems to vanish as well [2]. What happens then to the particle-antiparticle quantum degree of freedom? Obviously, we do not expect it to vanish. Quantum gravity, just as the ordinary quantum description of Nature, is believed to be complex. In fact, in any quantum approach the ' $i$ ' should enter the game through the quantum-mechanical position-momentum commutation relations, with position and momentum treated very symmetrically. The continuing presence of ' $i$ ' in the mathematical structure not involving explicit time variable suggests that it should be possible to interpret the existence of both particles and antiparticles also from the point of view of phase space alone, i.e. without an introduction of the concept of (external) time. Such an interpretation would shift our vantage point significantly, and could be useful in our search for a proper approach to (timeless) quantum gravity.

Keeping in mind that quantum mechanics 'lives' in phase space, I argued in a couple of recent papers (e.g. [12-15]) that viewing phase space as an arena of physics may provide a clue to 'space quantization'. At the level of basic assumptions, the relevant ideas differ from those of the formalism standardly known under the name of "phase-space quantization' (see e.g. [11]), however. Specifically, papers [12-15] consider a Dirac-like linearization of $\mathbf{x}^{2}+\mathbf{p}^{2}$, with position and momentum satisfying standard commutation relations. This leads to the appearance of a previously undiscussed discrete structure: the Clifford algebra of nonrelativistic phase space endowed with form $\mathbf{x}^{2}+\mathbf{p}^{2}$. This algebra is interesting because a part of it might be identified with the charge (isospin, hypercharge) structure of a single generation of elementary fermions in the Standard Model [5, 9]. In fact, the transition from nonrelativistic quantum phase space to its Clifford algebra may be regarded as a small step towards the quantization of 'space'. The main difference with respect to other approaches to quantum gravity is that here one deals with the phase space, not with the ordinary configuration space. In such a scheme, the spatial and internal quantum numbers of elementary particles are thought of as revealing the quantum layer underlying the geometry of phase space [15], considered as an arena of physics. This line of thinking leads to the idea that understanding the whole quantum structure manifested via the spectrum of elementary particles (in particular, their quantized masses and other parameters) should be essentially equivalent to understanding quantum gravity. An argument that the effects of quantum gravity should appear only when the Planck scale is reached is not really valid. As stressed by Kiefer [6], quantum gravity effects 'are not restricted to this scale a priori. The superposition principle allows the formation of non-trivial gravitational quantum states at any scale. (...) there may be situations where the quantum nature of gravity is visible-even far away from the Planck scale'. Kiefer then points out that such effects may be seen at cosmological scales, far beyond the scale of elementary particles. In fact, as long as we are dealing with quantum description, the issue of scale is simply irrelevant: quantum physics suggests that the familiar classical concepts of space and time are emergent concepts only, nonexisting (in the classical form) at the underlying quantum level. Aside from the above arguments, a 
more formal link to gravity seems to be present in the phase-space approach as well: when viewed from our 3D vantage point, the odd part of the Clifford algebra in question contains, besides scalars and vectors, also symmetric $S O(3)$ tensors of rank 2 [15].

In [12-14] various transformations between the lepton and quark sectors of the Clifford algebra were considered. In this note, I would like to discuss the meaning of these transformations and of their phase-space counterparts somewhat further, so that an understanding of the whole approach in terms of the concepts of positions and momenta may be deepened, including an interpretation of the relation between leptons, quarks, and their antiparticles in phase-space terms.

While our phase-space approach originates from the wish to treat momenta and positions in a more symmetric way [4], it is obvious that one cannot restore full symmetry between them: the momenta and positions would be then completely alike, which would be in conflict with the observed physical reality. In fact, putting aside the connection between the standard concept of mass and momentum (see [15]), an important difference between momenta and positions can be identified when one considers composite systems of ordinary particles (by ordinary particles I mean those particles which may be individually observed, like leptons, hadrons, and systems built thereof, but not quarks). For such systems, one observes that the physical momentum of the system is obtained by simply adding the physical momenta of its components, independently of where (in the classical case) the particles are. On the other hand, an (approximate) description of the physical position of the whole system is best provided by certain average of individual positions of its components (i.e., by their center of mass). Keeping in mind that one of our goals is to describe hadrons as composite systems made of quarks, it is natural to ensure that additivity of the just discussed or similar type be included in the description involving quarks. Towards the end of this paper we shall show that the rôle played by the additivity principle seems to be an important one: if the proposed ideas are basically correct, it is intimately related to quark unobservability and the emergence of mesons and baryons.

\section{Nonrelativistic Phase Space and Its Clifford Algebra}

\subsection{Phase Space}

Our approach is driven by the old wish to reduce dynamics to geometry, which motivates the search for a 'pregeometric algebra', a quantum-level counterpart of geometry. As already mentioned, this quantum-level algebra should be capable of relating to geometry such quantum features of Nature as the existence of internal quantum numbers and the quantization of particle masses. Thus, the corresponding effective quantum description in phase space should involve, besides the Planck constant, also a dimensional constant setting the mass scale. These constants together (plus the speed of light) determine an absolute scale of momenta and distances, and permit measuring the distances in units of momenta and vice versa.

For the above reasons, it was argued in [12] that a natural generalization of the $O(3)$ symmetry of ordinary 3D space to the case of nonrelativistic quantum phase space consists in considering the invariance of

$$
\mathbf{x}^{2}+\mathbf{p}^{2}
$$

(originally identified with Hamiltonian, but now viewed in geometrical terms), with $\mathbf{x}$ and $\mathbf{p}$ being the operators of physical positions and momenta, subject to the condition that

$$
\left[x_{k}, p_{l}\right]=i \delta_{k l}
$$


is also invariant. With the absolute scales of positions and momenta fixed, the symplectic rescalings admitted by (2) are obviously no longer allowed.

As is well known, the resulting symmetry is then $U(1) \otimes S U(3)$. Note that this symmetry is in fact present already in the strictly classical case, with Poisson brackets in place of the commutators.

A straightforward generalization of the set of $(1,2)$ is obtained by realizing that, since $i$ is defined only up to a sign, (2) may be replaced by

$$
\left[x_{k}, p_{l}\right]=-i \delta_{k l},
$$

leading to a different copy of the $U(1) \otimes S U(3)$ symmetry.

It should also be stressed that the operation of complex conjugation does not connect (2) with (3). Indeed, using the standard representation $p_{k}=-i \frac{d}{d x_{k}}$ one obtains that complex conjugation corresponds to

$$
i \rightarrow-i, \quad \mathbf{x} \rightarrow \mathbf{x}, \quad \mathbf{p} \rightarrow-\mathbf{p} .
$$

Under complex conjugation the set of $(1,2)$ (or alternatively, the set of $(1,3)$ ) remains therefore invariant.

\subsection{Clifford Algebra and Charges of Elementary Fermions}

Linearization of expression (1) à la Dirac (with commuting positions and momenta) leads to Clifford algebra built from the basic elements $A_{k}$ and $B_{l}$, associated with momentum $p_{k}$ and position $x_{l}$ respectively. We use the following explicit representation:

$$
\begin{aligned}
A_{k} & =\sigma_{k} \otimes \sigma_{0} \otimes \sigma_{1}, \\
B_{k} & =\sigma_{0} \otimes \sigma_{k} \otimes \sigma_{2}, \\
B_{7} \equiv i A_{1} A_{2} A_{3} B_{1} B_{2} B_{3} & =\sigma_{0} \otimes \sigma_{0} \otimes \sigma_{3},
\end{aligned}
$$

where $B_{7}$ is the 7 th anticommuting element of the algebra. ${ }^{1}$

In [12] it was shown that when the position-momentum commutation relations are accepted, one obtains

$$
R^{t o t} \equiv(\mathbf{A} \cdot \mathbf{p}+\mathbf{B} \cdot \mathbf{x})(\mathbf{A} \cdot \mathbf{p}+\mathbf{B} \cdot \mathbf{x})=\left(\mathbf{p}^{2}+\mathbf{x}^{2}\right)-\frac{i}{2}\left[A_{k}, B_{k}\right],
$$

(summation over repeated indices implied) where the last term comes about because $x_{k}$ and $p_{k}$ do not commute. In [12] it was then furthermore proposed that electric charge $Q$ is equal to operator $\frac{1}{6} R^{\text {tot }} B_{7}$, evaluated for the lowest level of $\mathbf{p}^{2}+\mathbf{x}^{2}$, i.e.

$$
Q=\frac{1}{6}\left(\left(\mathbf{p}^{2}+\mathbf{x}^{2}\right)_{\text {lowest }}-\frac{i}{2}\left[A_{k}, B_{k}\right]\right) B_{7} \equiv I_{3}+\frac{Y}{2},
$$

with (weak) isospin $I_{3}$ and (weak) hypercharge $Y$ given by

$$
I_{3}=\frac{B_{7}}{2}
$$

\footnotetext{
${ }^{1}$ The product $\Pi_{k=1}^{3} A_{k} B_{k}$ is the $6 \mathrm{D}$ generalization of $A_{1} B_{1}$ relevant for the $2 \mathrm{D}$ phase space. For the latter case, an identification of such a product with the imaginary unit was proposed in [7].
} 


$$
Y=-\frac{i}{6}\left[A_{k}, B_{k}\right] B_{7}=\frac{1}{3} \sigma_{k} \otimes \sigma_{k} \otimes \sigma_{0} \equiv \sum_{k} Y_{k}
$$

The eigenvalues of $I_{3}$ are $\pm 1 / 2$, those of 'partial hypercharges' $Y_{k}$ are $\pm 1 / 3$, while for the hypercharge $Y$ we have $-1,+1 / 3,+1 / 3,+1 / 3$, corresponding to a lepton and a triplet of quarks respectively. Equation (7) yields then the charges of all eight particles of a single generation of the Standard Model.

\section{Isospin Reversal and Charge Conjugation}

Transformations in the phase space and in its Clifford algebra are related by the requirement of the invariance of $\mathbf{A} \cdot \mathbf{p}+\mathbf{B} \cdot \mathbf{x}$. Below we shall use this invariance to provide phasespace interpretations of the connections between the particles of a single Standard Model generation.

\subsection{Isospin Reversal}

Consider the following reflection in the algebraic counterpart of phase space:

$$
\mathbf{A}^{\prime}=I_{1} \mathbf{A} I_{1}^{-1}=\mathbf{A}, \quad \mathbf{B}^{\prime}=I_{1} \mathbf{B} I_{1}^{-1}=-\mathbf{B},
$$

where $I_{n}=\sigma_{0} \otimes \sigma_{0} \otimes \sigma_{n}$. Under the above operation, we have $B_{7} \rightarrow-B_{7}, i \rightarrow i$, and, consequently, this transformation swaps $I_{3}= \pm \frac{1}{2}$ sectors, while keeping hypercharge unchanged:

$$
I_{3}^{\prime}=-I_{3}, \quad Y^{\prime}=Y .
$$

Upon requiring the invariance of the expression $\mathbf{A} \cdot \mathbf{p}+\mathbf{B} \cdot \mathbf{x}$ one finds that (9) corresponds to the following transformation in phase space:

$$
\mathbf{p} \rightarrow \mathbf{p}^{\prime}=\mathbf{p}, \quad \mathbf{x} \rightarrow \mathbf{x}^{\prime}=-\mathbf{x}
$$

Alternatively, one might consider $\mathbf{A}^{\prime}=I_{2} \mathbf{A} I_{2}^{-1}=-\mathbf{A}, \mathbf{B}^{\prime}=I_{2} \mathbf{B} I_{2}^{-1}=\mathbf{B}$, and $\mathbf{p}^{\prime}=-\mathbf{p}$, $\mathbf{x}^{\prime}=\mathbf{x}$, which is related to (9) via ordinary 3D reflection. Then, (10) still holds. Both types of reflection correspond to the transformation

$$
\left[x_{k}, p_{l}\right]=i \delta_{k l} \rightarrow\left[x_{k}, p_{l}\right]=-i \delta_{k l},
$$

with $\mathbf{p}^{2}+\mathbf{x}^{2}$ unchanged, i.e. we recover the other copy of $U(1) \otimes S U(3)$ (cf. (3)). We prefer to work with phase-space reflection defined in (9) as it does not affect the momentum of the particle under consideration.

If lepton $L$ of isospin $I_{3}=+1 / 2$ corresponds to the following division of the six basic elements of Clifford algebra into the counterparts of canonical momenta, and counterparts of canonical positions:

$$
\left(\mathbf{A}^{L}, \mathbf{B}^{L}\right)=(\mathbf{A}, \mathbf{B}),
$$

with (the operators of) canonical momenta $\mathbf{p}^{L}$ (positions $\mathbf{x}^{L}$ ) identified with (the operators of) physical momenta $\mathbf{p}$ (positions $\mathbf{x}$ ), i.e.:

$$
\left(\mathbf{p}^{L}, \mathbf{x}^{L}\right)=(\mathbf{p}, \mathbf{x})
$$


then its partner $L^{\prime}$ of isospin $I_{3}=-1 / 2$ corresponds to

$$
\left(\mathbf{A}^{L^{\prime}}, \mathbf{B}^{L^{\prime}}\right)=(\mathbf{A},-\mathbf{B}),
$$

and

$$
\left(\mathbf{p}^{L^{\prime}}, \mathbf{x}^{L^{\prime}}\right)=(\mathbf{p},-\mathbf{x})
$$

The choice of (9) for the representation of isospin reversal ensures that the addition of the canonical momenta of leptons is equivalent to the addition of their physical momenta, independently of their eigenvalues of $I_{3}$.

\subsection{Charge Conjugation}

As is well known, charge conjugation is closely related to complex conjugation. A possible corresponding operation in phase space was given in (4). As in the case of phase-space reflection we prefer to keep the momentum unchanged (so that we go from a particle of a given physical momentum to its antiparticle of the same physical momentum) and define the operation of charge conjugation in phase space via:

$$
\overline{\mathbf{p}}=\mathbf{p}, \quad \overline{\mathbf{x}}=-\mathbf{x}, \quad \bar{i}=i^{*}=-i,
$$

as is also obtained from the straightforward application of complex conjugation if one takes $x_{k}=i \frac{d}{d p_{k}}$, with $p_{k}$ real. As before, the reason for this choice of the definition of charge conjugation is that the addition of physical momenta of particles and antiparticles is now straightforward: one does not have to employ any specific method of inverting the signs of antiparticle momentum variables. In Clifford algebra the operation of charge conjugation should be obtained from $i \rightarrow i^{*}=-i$ through:

$$
\mathbf{A} \rightarrow \mathbf{A}^{*}, \quad \mathbf{B} \rightarrow \mathbf{B}^{*}
$$

Then, we get

$$
\mathbf{A} \cdot \mathbf{p}+\mathbf{B} \cdot \mathbf{x} \rightarrow \mathbf{A}^{*} \cdot \mathbf{p}-\mathbf{B}^{*} \cdot \mathbf{x}
$$

The condition of the invariance of $\mathbf{A} \cdot \mathbf{p}+\mathbf{B} \cdot \mathbf{x}$ under charge conjugation may be satisfied if it is possible to find a unitary transformation such that $\mathbf{A}^{*} \rightarrow \mathbf{A}, \mathbf{B}^{*} \rightarrow-\mathbf{B}$, i.e. such $C$ that:

$$
\begin{aligned}
& \overline{\mathbf{A}}=C \mathbf{A}^{*} C^{-1}=\mathbf{A}, \\
& \overline{\mathbf{B}}=C \mathbf{B}^{*} C^{-1}=-\mathbf{B},
\end{aligned}
$$

so that a counterpart of (17) is obtained:

$$
\overline{\mathbf{A}}=\mathbf{A}, \quad \overline{\mathbf{B}}=-\mathbf{B}, \quad\left(\text { and } \bar{i}=i^{*}=-i\right) .
$$

One may check that

$$
C=C^{-1}=\sigma_{2} \otimes \sigma_{2} \otimes \sigma_{3} .
$$


possesses the required property. ${ }^{2}$ From (5) one then finds that $B_{7} \rightarrow B_{7}$, whence

$$
I_{3} \rightarrow I_{3}, \quad Y \rightarrow Y
$$

Thus, the exponent in $U=\exp (i Q)$ changes as follows

$$
i Q=i\left(I_{3}+Y / 2\right) \rightarrow-i\left(I_{3}+Y / 2\right) \stackrel{\text { def }}{=} i \bar{Q}=i\left(\bar{I}_{3}+\bar{Y} / 2\right),
$$

i.e. the antiparticles have opposite charges, isospins and hypercharges:

$$
\bar{Q}=-Q, \quad \bar{I}_{3}=-I_{3}, \quad \bar{Y}=-Y .
$$

The antiparticle to any lepton $L$ of isospin $I_{3}=+1 / 2$, i.e. an antilepton $\bar{L}$ of isospin $\bar{I}_{3}=-1 / 2$, corresponds to

$$
\left(\mathbf{A}^{\bar{L}}, \mathbf{B}^{\bar{L}}, i^{*}\right)=(\mathbf{A},-\mathbf{B},-i)
$$

and

$$
\left(\mathbf{p}^{\bar{L}}, \mathbf{x}^{\bar{L}}\right)=(\mathbf{p},-\mathbf{x}),
$$

where $\mathbf{p}$ and $\mathbf{x}$ are the (operators of) physical momenta and positions of an antiparticle. Thus, apart from $i \rightarrow-i$, the difference between particles and antiparticles amounts again to a reflection in position space. We stress that the difference between the phase space reflection of $(9,11)$ and the representation $(17,21)$ of charge conjugation in phase space is tiny: it is just the change of the sign in front of free-standing $i$, without affecting $\mathbf{p}$ or $\mathbf{x}$. The $\bar{I}_{3}=$ $+1 / 2$ isospin partner $\bar{L}^{\prime}$ of antilepton $\bar{L}$ (of $(26,27)$ ) corresponds to

$$
\begin{aligned}
\left(\mathbf{A}^{\bar{L}^{\prime}}, \mathbf{B}^{\bar{L}^{\prime}}, i^{*}\right) & =(\mathbf{A}, \mathbf{B},-i), \\
\left(\mathbf{p}^{\bar{L}^{\prime}}, \mathbf{x}^{\bar{L}^{\prime}}\right) & =(\mathbf{p}, \mathbf{x}) .
\end{aligned}
$$

With the above definitions, the additivity of the canonical momenta of leptons and/or antileptons is equivalent to the additivity of their physical momenta, irrespectively of whether we are dealing with particles, antiparticles, or both particles and antiparticles, and irrespectively of their values of $I_{3}$.

\section{Lepton-Quark Transformations}

Apart from the just discussed discrete transformations corresponding to isospin reversal and charge conjugation, one may consider lepton-quark transformations. As shown in [12-14], the transformations from the lepton sector (of $Y=-1$ ) to the three quark sectors (defined by the sets of the eigenvalues $\pm 1 / 3$ of $Y_{k}$ 's such that $Y=\sum_{k} Y_{k}=+1 / 3$ ) correspond to specific cases of those phase-space $S O(6)$ transformations which go beyond $U(1) \otimes S U(3)$

\footnotetext{
${ }^{2}$ In [12] the operation of charge conjugation was defined in a similar way, but with a different $C$, proportional to $\sigma_{2} \otimes \sigma_{2} \otimes \sigma_{2}$. I now find the latter expression inadequate for a couple of reasons, the requirement of the invariance of $\mathbf{A} \cdot \mathbf{p}+\mathbf{B} \cdot \mathbf{x}$ among them. Adopting prescription (22) changes the sign with which $\mathbf{B}$ (and $\left.B_{7}\right)$ transforms under charge conjugation (when compared to [12]), but does not affect the conclusions of [13, 14] since the eigenvalues of $B_{7}$ and $-B_{7}$ are identical.
} 
(and the standard 3D rotations in particular). Among these 'genuine' $S O(6)$ transformations there are, in particular, the following (pairs of) rotations:

$$
\begin{array}{ll}
\tilde{p}_{1}=p_{1} \cos \phi+x_{3} \sin \phi, & \tilde{x}_{1}=x_{1} \cos \phi+p_{3} \sin \phi, \\
\tilde{x}_{3}=x_{3} \cos \phi-p_{1} \sin \phi, & \tilde{p}_{3}=p_{3} \cos \phi-x_{1} \sin \phi,
\end{array}
$$

which leave $\left(x_{2}, p_{2}\right)$ unchanged: $\tilde{p}_{2}=p_{2}, \tilde{x}_{2}=x_{2}$. Transformation between the lepton sector and the colour-2 quark sector is obtained when a specific condition is imposed on (30). This condition amounts to the requirement that the position-momentum commutation relations in new variables, i.e.

$$
\left[\tilde{x}_{k}, \tilde{p}_{l}\right]=i \Delta_{k l}
$$

be diagonal, and that new canonical positions $\tilde{x}_{k}$ (and new canonical momenta $\tilde{p}_{k}$ ) commute among themselves. As discussed in [12], a nontrivial case (corresponding to a quark of colour-2) is obtained for $\phi= \pm \pi / 2$, which yields

$$
\Delta=\left[\begin{array}{ccc}
-1 & 0 & 0 \\
0 & 1 & 0 \\
0 & 0 & -1
\end{array}\right],
$$

with similar formulas for the two analogs of (30) corresponding to (pairs of) rotations leaving $\left(x_{3}, p_{3}\right)$ and $\left(x_{1}, p_{1}\right)$ unchanged. Equations (30) for $\phi= \pm \pi / 2$ define what we mean by canonical momenta and positions for quarks of colour 2 (up to a redefinition corresponding to $\phi=\pi / 2 \leftrightarrow \phi=-\pi / 2$, see later).

Putting aside the above three types of transformations (which interchange positions and momenta) and the nine types of transformations corresponding to $U(1) \otimes S U(3)$, one is left with the three remaining types of $S O(6)$ transformations. These are similar to ordinary $3 \mathrm{D}$ rotations, the difference being that rotations in momentum and position spaces are now performed in opposite senses:

$$
\begin{array}{ll}
\tilde{p}_{1}=p_{1} \cos \phi-p_{3} \sin \phi, & \tilde{x}_{1}=x_{1} \cos \phi+x_{3} \sin \phi, \\
\tilde{p}_{3}=p_{3} \cos \phi+p_{1} \sin \phi, & \tilde{x}_{3}=x_{3} \cos \phi-x_{1} \sin \phi,
\end{array}
$$

with $p_{2}, x_{2}$ unchanged. The diagonality condition again requires $\phi= \pm \pi / 2$. In both cases, therefore, only specific discrete cases of the 'genuine' phase-space rotations are allowed.

Phase-space transformations of $(30,33)$ have their obvious counterparts in Clifford algebra. Thus, as discussed in [12], the set of all discrete transformations, leading from any algebra element $Z$ to its colour- $n$ quark counterpart $Z^{Q n}$, consists of the following four alternatives (no summation over underlined indices):

$$
Z^{Q n}=\left\{\begin{array}{l}
\mathcal{R}_{0 \underline{n}, \pm} Z \mathcal{R}_{0 \underline{n}, \pm}^{-1}, \\
\mathcal{R}_{0 \underline{n}, \pm}^{\dagger} Z\left(\mathcal{R}_{0 \underline{n}, \pm}^{\dagger}\right)^{-1},
\end{array}\right.
$$

with

$$
\begin{aligned}
& \mathcal{R}_{0 n, \pm}=\left.\exp \left(i \phi F_{ \pm n}\right)\right|_{\phi=\pi / 2}=\exp \left(i \frac{\pi}{2} F_{ \pm n}\right)=1+i F_{ \pm n}-\left(F_{ \pm n}\right)^{2} \\
& \mathcal{R}_{0 n, \pm}^{\dagger}=\left.\exp \left(i \phi F_{ \pm n}\right)\right|_{\phi=-\pi / 2}=\exp \left(-i \frac{\pi}{2} F_{ \pm n}\right)=1-i F_{ \pm n}-\left(F_{ \pm n}\right)^{2}
\end{aligned}
$$


generated by the 'genuine' $S O(6) / S U(4)$ generators:

$$
\begin{aligned}
& F_{+n}=-\frac{i}{4} \epsilon_{n k l}\left[A_{k}, B_{l}\right]=\frac{1}{2} \epsilon_{n k l} \sigma_{k} \otimes \sigma_{l} \otimes \sigma_{3} \\
& F_{-n}=-\frac{i}{4} \epsilon_{n k l}\left(B_{k} B_{l}-A_{k} A_{l}\right)=\frac{1}{2}\left(\sigma_{0} \otimes \sigma_{n}-\sigma_{n} \otimes \sigma_{0}\right) \otimes \sigma_{0},
\end{aligned}
$$

corresponding (for $n=2$ ) to (30) and (33) respectively, and satisfying $\left(F_{ \pm n}\right)^{3}=F_{ \pm n}$. The four alternatives of (34) exist because operators $Y$ and $I_{3}$ are even in $A_{k}, B_{l}$, and therefore different transformations of $A_{k}, B_{l}$ may lead to the same result for $Y$ and $I_{3}$. In particular, for a given $n$, all four transformations (34) change the partial hypercharges $Y_{k}$ (and the isospin $I_{3}$ ) in exactly the same way, which amounts to interchanging the $Y=-1$ (lepton) sector with the $Y=+1 / 3$ (quark) colour- $n$ sector. (For example, for $\mathcal{R}_{0 n,+}$ and $\mathcal{R}_{0 n,+}^{(\dagger)}$ this may be seen from $(8,37,39)$.)

When describing all three colours simultaneously, one might in principle consider various combinations of the $\mathcal{R}_{0 n, \pm} \pm^{-}$and $\mathcal{R}_{0 n, \pm}^{\dagger}$-induced transformations, taking any of the four options in (34) for a given colour (e.g. choosing either the set $\left\{\mathcal{R}_{01,+}, \mathcal{R}_{02,+}, \mathcal{R}_{03,+}\right\}$, or the set $\left\{\mathcal{R}_{01,+}, \mathcal{R}_{02,-}, \mathcal{R}_{03,+}^{\dagger}\right\}$, or..., etc.). Full symmetry between the three directions for both position and momentum, as observed in our 3D world, requires however that we admit only such combinations which do not depend on our way of labelling the three directions with numbers 1, 2, 3 (because the assignment of labels of, say, a right-handed system of coordinates to the three, yet unlabelled, directions of our ordinary 3D space is arbitrary). This restricts our study to four sets of transformations only, each set specified by the sense of the rotation by $\pi / 2$ (i.e. $\phi= \pm \pi / 2$ ), and by the type of $S O(6) / S U(4)$ generator used (either $F_{+n}$ or $\left.F_{-n}\right)$.

Below we will consider the action of the transformations of (34) upon the algebraic counterparts of momenta and positions, i.e. upon elements $A_{k}$ and $B_{l}$. We start with the set of $\mathcal{R}_{0 n,+}$-induced transformations.

\section{$4.1 \mathcal{R}_{0 n,+}$-induced Transformations}

The $\mathcal{R}_{0 n,+}$-induced $(\phi=+\pi / 2)$ transformations of $A_{k}$ and $B_{l}$ were evaluated in [15] to be:

$$
\begin{aligned}
& A_{k}^{Q n}=\mathcal{R}_{0 \underline{n},+} A_{k} \mathcal{R}_{0 \underline{n},+}^{-1}=\delta_{\underline{n k}} A_{\underline{n}}-\epsilon_{n k m} B_{m}, \\
& B_{k}^{Q n}=\mathcal{R}_{0 \underline{n},+} B_{k} \mathcal{R}_{0 \underline{n},+}^{-1}=\delta_{\underline{n} k} B_{\underline{n}}-\epsilon_{n k m} A_{m} .
\end{aligned}
$$

If we start with lepton $L$ of $I_{3}=+1 / 2$, i.e. with the counterparts of canonical momenta being $\left(\mathbf{A}^{L}, \mathbf{B}^{L}\right)=(\mathbf{A}, \mathbf{B})$, the above formulas yield their coloured quark counterparts $\left(\mathbf{A}^{Q n}, \mathbf{B}^{Q n}\right)=\left(\left(A_{1}^{Q n}, A_{2}^{Q n}, A_{3}^{Q n}\right),\left(B_{1}^{Q n}, B_{2}^{Q n}, B_{3}^{Q n}\right)\right)$. The relevant expressions may be written in a transparent matrix form:

$$
\begin{gathered}
A^{Q} \equiv\left[\begin{array}{l}
\mathbf{A}^{Q 1} \\
\mathbf{A}^{Q 2} \\
\mathbf{A}^{Q 3}
\end{array}\right]=\left[\begin{array}{ccc}
A_{1} & -B_{3} & +B_{2} \\
+B_{3} & A_{2} & -B_{1} \\
-B_{2} & +B_{1} & A_{3}
\end{array}\right], \\
B^{Q} \equiv\left[\begin{array}{l}
\mathbf{B}^{Q 1} \\
\mathbf{B}^{Q 2} \\
\mathbf{B}^{Q 3}
\end{array}\right]=\left[\begin{array}{ccc}
B_{1} & -A_{3} & +A_{2} \\
+A_{3} & B_{2} & -A_{1} \\
-A_{2} & +A_{1} & B_{3}
\end{array}\right],
\end{gathered}
$$


with Clifford algebra counterparts $\mathbf{A}^{Q n}$ for canonical momenta and $\mathbf{B}^{Q n}$ for canonical positions gathered in row $n$ for the sector of colour $n$. The imaginary unit $i$ is of course unchanged as $\mathcal{R}_{0 \underline{n},+} i \mathcal{R}_{0 n,+}^{-1}=i$ for any $n$. Since $i$ is unaffected, the pair of matrices $\left(A^{Q}\right.$, $B^{Q}$ ) must correspond to quarks. Furthermore, since $\mathcal{R}_{0 \underline{n},+} I_{3} \mathcal{R}_{0 \underline{n},+}^{-1}=I_{3}$, again for any $n$, our quarks still have $I_{3}=+1 / 2$.

\section{$4.2 \mathcal{R}_{0 n,+}^{\dagger}$-induced Transformations}

The $\mathcal{R}_{0 n,+}^{\dagger}$-induced $(\phi=-\pi / 2)$ transformations of $A_{k}$ and $B_{l}$ were found in [15] to be:

$$
\begin{aligned}
& A_{k}^{Q n(\dagger)}=\mathcal{R}_{0 \underline{n},+}^{\dagger} A_{k}\left(\mathcal{R}_{0 \underline{n},+}^{\dagger}\right)^{-1}=\delta_{\underline{n} k} A_{\underline{n}}+\epsilon_{n k m} B_{m}, \\
& B_{k}^{Q n(\dagger)}=\mathcal{R}_{0 \underline{n},+}^{\dagger} B_{k}\left(\mathcal{R}_{0 \underline{n},+}^{\dagger}\right)^{-1}=\delta_{\underline{n} k} B_{\underline{n}}+\epsilon_{n k m} A_{m} .
\end{aligned}
$$

If we start again with lepton $L$ of isospin $I_{3}=+1 / 2$, i.e. with the counterparts of canonical momenta being $\left(\mathbf{A}^{L}, \mathbf{B}^{L}\right)=(\mathbf{A}, \mathbf{B})$, the above formulas yield their coloured quark counterparts $\left(\mathbf{A}^{Q n(\dagger)}, \mathbf{B}^{Q n(\dagger)}\right)$. The corresponding matrices of the Clifford algebra counterparts of canonical momenta and canonical positions are then:

$$
\begin{aligned}
A^{Q(\dagger)} & \equiv\left[\begin{array}{l}
\mathbf{A}^{Q 1(\dagger)} \\
\mathbf{A}^{Q 2(\dagger)} \\
\mathbf{A}^{Q 3(\dagger)}
\end{array}\right]=\left[\begin{array}{ccc}
A_{1} & +B_{3} & -B_{2} \\
-B_{3} & A_{2} & +B_{1} \\
+B_{2} & -B_{1} & A_{3}
\end{array}\right], \\
B^{Q(\dagger)} & \equiv\left[\begin{array}{l}
\mathbf{B}^{Q 1(\dagger)} \\
\mathbf{B}^{Q 2(\dagger)} \\
\mathbf{B}^{Q 3(\dagger)}
\end{array}\right]=\left[\begin{array}{ccc}
B_{1} & +A_{3} & -A_{2} \\
-A_{3} & B_{2} & +A_{1} \\
+A_{2} & -A_{1} & B_{3}
\end{array}\right] .
\end{aligned}
$$

Since, as in the previous case, $i$ and $I_{3}$ are unaffected, the pair of matrices $\left(A^{Q(\dagger)}, B^{Q(\dagger)}\right)$ just as the pair $\left(A^{Q}, B^{Q}\right)$-must correspond to quarks of $I_{3}=+1 / 2$.

As discussed in [12-15] and seen above, the $F_{+n}$-induced transformations lead to some of the $A_{k}$ 's being replaced by some of the $B_{l}$ 's, while their phase-space counterparts, i.e. the specific cases of transformations (30) (or their analogs) lead to some of physical position variables playing the rôle of canonical momenta.

\section{$4.3 \mathcal{R}_{0 n,-}-$ and $\mathcal{R}_{0 n,-}^{\dagger}$-induced Transformations}

As discussed in [15], for any fixed $n$ the $\mathcal{R}_{0 n,-}$-induced transformations are related to the $\mathcal{R}_{0 n,+}$ via specific $U(1) \otimes S U(3)$ transformations (with similar statement holding also for $\mathcal{R}_{0 n,-}^{\dagger}$ and $\mathcal{R}_{0 n,+}^{\dagger}$ ). The $\mathcal{R}_{0 n,-}$ ( and $\mathcal{R}_{0 n,-}^{\dagger}$ ) induced transformations do not swap some $A_{k}$ 's with some of the $B_{l}$ 's, but just rotate A's and B's in the opposite senses. When the ordinary $3 \mathrm{D}$ rotations are also allowed, the effect of $\mathcal{R}_{0 n,-}$ - and $\mathcal{R}_{0 n,-}^{\dagger}$-induced transformations is equivalent to admitting appropriate transformations in the space of canonical positions only. Thus, they do not really bring in more symmetry between the momentum and position coordinates that Max Born wanted so badly [4]. Consequently, they are of no interest to us here.

\section{Representations}

For a fixed $n$ (take $n=1)$ we have:

$$
\mathbf{A}^{Q 1}=\left(A_{1},-B_{3},+B_{2}\right), \quad \mathbf{B}^{Q 1}=\left(B_{1},-A_{3},+A_{2}\right),
$$




$$
\mathbf{A}^{Q 1(\dagger)}=\left(A_{1},+B_{3},-B_{2}\right), \quad \mathbf{B}^{Q 1(\dagger)}=\left(B_{1},+A_{3},-A_{2}\right) .
$$

The above two possibilities for the counterparts of canonical momenta and positions for quark of colour 1 are related by an ordinary 3D rotation by $\pi$ around the 1 st axis in both $\mathbf{A}$ and $\mathbf{B}$ spaces (alternatively, the connection may be provided by appropriate reflections: $\mathbf{B} \rightarrow-\mathbf{B}$ for $A^{Q}$, and $\mathbf{A} \rightarrow-\mathbf{A}$ for $B^{Q}$ ).

The corresponding canonical momenta and positions are

$$
\begin{array}{ll}
\mathbf{p}^{Q 1}=\left(p_{1},-x_{3},+x_{2}\right), & \mathbf{x}^{Q 1}=\left(x_{1},-p_{3},+p_{2}\right), \\
\mathbf{p}^{Q 1(\dagger)}=\left(p_{1},+x_{3},-x_{2}\right), & \mathbf{x}^{Q 1(\dagger)}=\left(x_{1},+p_{3},-p_{2}\right) .
\end{array}
$$

As mentioned before, as long as $i$ is unchanged, both $\left(\mathbf{A}^{Q 1}, \mathbf{B}^{Q 1}\right)$ and $\left(\mathbf{A}^{Q 1(\dagger)}, \mathbf{B}^{Q 1(\dagger)}\right)$ represent algebraic counterparts of canonical variables for quark of colour 1 and isospin $I_{3}=+1 / 2$. We now choose the first one of the two forms above (i.e. the first rows in (41, 42)) to represent quark of isospin $I_{3}=+1 / 2$ and colour 1 . For arbitrary colour $n$, we thus have

$$
\begin{aligned}
& \mathbf{A}^{Q n}=\mathcal{R}_{0 \underline{n},+} \mathbf{A}^{L} \mathcal{R}_{0 \underline{n},+}^{-1}, \\
& \mathbf{B}^{Q n}=\mathcal{R}_{0 \underline{n,+}} \mathbf{B}^{L} \mathcal{R}_{0 \underline{n,+}}^{-1} .
\end{aligned}
$$

The choice between the sets of $\mathcal{R}_{0 n,+}$ and $\mathcal{R}_{0 n,+}^{\dagger}$-induced transformations is arbitrary, but once it is done, it has to be strictly adhered to. The situation is analogous to a choice between $\left(p_{1},-p_{3},+p_{2}\right)$, obtained from $\left(p_{1}, p_{2}, p_{3}\right)$ through a rotation by $\pi / 2$ around the first axis, and $\left(p_{1},+p_{3},-p_{2}\right)$, obtained through a similar rotation by $-\pi / 2$. Each one of these two representations (or, in this case, also the original one, i.e. $\left.\left(p_{1}, p_{2}, p_{3}\right)\right)$ may be used. The condition that the additivity of the momenta of different particles be properly taken care of requires, however, that only one such form of description is universally chosen for all particles. For example, for the first convention, the total momentum of a system of two particles is properly calculated via the addition of the corresponding representatives:

$$
\left(p_{1}^{(1)},-p_{3}^{(1)},+p_{2}^{(1)}\right)+\left(p_{1}^{(2)},-p_{3}^{(2)},+p_{2}^{(2)}\right)=\left(p_{1}^{\text {tot }},-p_{3}^{\text {tot }},+p_{2}^{\text {tot }}\right) .
$$

\subsection{Quarks}

For the convention of (43) and with the explicit forms of $\mathbf{A}^{Q n}$ and $\mathbf{B}^{Q n}$ given in (38), the matrices of the canonical momenta and positions for quarks of isospin $I_{3}=+1 / 2$ are, by analogy:

$$
\begin{gathered}
P^{Q} \equiv\left[\begin{array}{l}
\mathbf{p}^{Q 1} \\
\mathbf{p}^{Q 2} \\
\mathbf{p}^{Q 3}
\end{array}\right]=\left[\begin{array}{ccc}
p_{1}^{1} & -x_{3}^{1} & +x_{2}^{1} \\
+x_{3}^{2} & p_{2}^{2} & -x_{1}^{2} \\
-x_{2}^{3} & +x_{1}^{3} & p_{3}^{3}
\end{array}\right], \\
X^{Q} \equiv\left[\begin{array}{l}
\mathbf{x}^{Q 1} \\
\mathbf{x}^{Q 2} \\
\mathbf{x}^{Q 3}
\end{array}\right]=\left[\begin{array}{ccc}
x_{1}^{1} & -p_{3}^{1} & +p_{2}^{1} \\
+p_{3}^{2} & x_{2}^{2} & -p_{1}^{2} \\
-p_{2}^{3} & +p_{1}^{3} & x_{3}^{3}
\end{array}\right],
\end{gathered}
$$

where we have allowed that the expressions on the right, containing the physical momenta and positions of quarks of different colours, in general may depend on quark colour (hence superscript $n$ for row $n$ ). 
Transition to the sector of $I_{3}=-1 / 2$ quarks is obtained from (43) by the action of $\mathcal{R}_{0 \underline{n},+^{-}}$ induced transformation upon $\left(\mathbf{A}^{L^{\prime}}, \mathbf{B}^{L^{\prime}}\right)=(\mathbf{A},-\mathbf{B})$, corresponding to lepton $L^{\prime}$ of isospin $I_{3}=-1 / 2$ :

$$
\begin{aligned}
& \mathbf{A}^{Q^{\prime} n}=\mathcal{R}_{0 \underline{n,},+} \mathbf{A}^{L^{\prime}} \mathcal{R}_{0 \underline{n,+}}^{-1}=\mathcal{R}_{0 \underline{n,+}} \mathbf{A} \mathcal{R}_{0 \underline{n},+}^{-1}, \\
& \mathbf{B}^{Q^{\prime} n}=\mathcal{R}_{0 \underline{n},+} \mathbf{B}^{L^{\prime}} \mathcal{R}_{0 \underline{n},+}^{-1}=\mathcal{R}_{0 \underline{n},+}(-\mathbf{B}) \mathcal{R}_{0 \underline{n},+}^{-1} .
\end{aligned}
$$

Thus,

$$
\begin{aligned}
& A^{Q^{\prime}}=\left[\begin{array}{ccc}
A_{1} & -B_{3} & +B_{2} \\
+B_{3} & A_{2} & -B_{1} \\
-B_{2} & +B_{1} & A_{3}
\end{array}\right]=A^{Q}, \\
& B^{Q^{\prime}}=-\left[\begin{array}{ccc}
B_{1} & -A_{3} & +A_{2} \\
+A_{3} & B_{2} & -A_{1} \\
-A_{2} & +A_{1} & B_{3}
\end{array}\right]=-B^{Q} .
\end{aligned}
$$

The corresponding matrices of the canonical momenta and positions for quarks of isospin $I_{3}=-1 / 2$ are

$$
\begin{aligned}
P^{Q^{\prime}}= & {\left[\begin{array}{ccc}
p_{1}^{1} & -x_{3}^{1} & +x_{2}^{1} \\
+x_{3}^{2} & p_{2}^{2} & -x_{1}^{2} \\
-x_{2}^{3} & +x_{1}^{3} & p_{3}^{3}
\end{array}\right]=P^{Q}, } \\
X^{Q^{\prime}} & =-\left[\begin{array}{ccc}
x_{1}^{1} & -p_{3}^{1} & +p_{2}^{1} \\
+p_{3}^{2} & x_{2}^{2} & -p_{1}^{2} \\
-p_{2}^{3} & +p_{1}^{3} & x_{3}^{3}
\end{array}\right]=-X^{Q},
\end{aligned}
$$

where, as before, we have allowed that the expressions in the middle, containing the physical momenta and positions of quarks of different colours, in general may depend on quark colour. On the other hand, for better transparency of the comparison of the $I_{3}=-1 / 2$ and $I_{3}=+1 / 2$ sectors, we have suppressed the superscript 'prime' on physical variables.

Applying the operation of phase-space reflection defined in (9) to the set $\left[\left(A^{Q}, B^{Q}\right)\right.$, $\left.\left(A^{Q^{\prime}}, B^{Q^{\prime}}\right)\right]$ (corresponding to $\left.\left[I_{3}=+1 / 2, I_{3}=-1 / 2\right]\right)$ yields

$$
\begin{gathered}
I_{1}\left(A^{Q}, B^{Q}\right) I_{1}^{-1}=\left(A^{Q^{\prime}(\dagger)}, B^{Q^{\prime}(\dagger)}\right), \\
I_{1}\left(A^{Q^{\prime}}, B^{Q^{\prime}}\right) I_{1}^{-1}=\left(A^{Q(\dagger)}, B^{Q(\dagger)}\right),
\end{gathered}
$$

i.e. one obtains representatives for quarks with interchanged eigenvalues of isospin $( \pm 1 / 2 \rightarrow \mp 1 / 2)$, but in the other representation. This is so because $I_{1} \mathcal{R}_{0 n,+} I_{1}^{-1}=\mathcal{R}_{0 n,+}^{(\dagger)}$, and is related to a change from a left- to a right-handed labelling of the system of coordinates.

To summarize: just as leptons of isospin $I_{3}= \pm 1 / 2$ correspond to $\left(\mathbf{A}^{L}, \pm \mathbf{B}^{L}\right)$ and $\left(\mathbf{p}^{L}, \pm \mathbf{x}^{L}\right)$, so do quarks of isospin $I_{3}= \pm 1 / 2$ correspond to $\left(A^{Q}, \pm B^{Q}\right)$ and $\left(P^{Q}, \pm X^{Q}\right)$. Note that the forms of the canonical momenta $\left(P^{Q}\right)$ are identical for both isospins.

\subsection{Antiquarks}

We now recall that the transition to antiparticles is obtained via complex conjugation of (18). In a lepton sector, this leads to $\left(\mathbf{A}^{\mathbf{L}}, \mathbf{B}^{L}, i\right)=(\mathbf{A}, \mathbf{B}, i) \rightarrow\left(\mathbf{A}^{\bar{L}}, \mathbf{B}^{\bar{L}}, i^{*}\right)=(\mathbf{A},-\mathbf{B},-i)$ and 
$\left(\mathbf{p}^{\mathbf{L}}, \mathbf{x}^{L}\right)=(\mathbf{p}, \mathbf{x}) \rightarrow\left(\mathbf{p}^{\bar{L}}, \mathbf{x}^{\bar{L}}\right)=(\mathbf{p},-\mathbf{x})$. In the quark sector, this leads to the following matrices for the counterparts of canonical momenta and positions of antiquarks:

(1) for the $\bar{I}_{3}=-1 / 2$ antiparticles of the $I_{3}=+1 / 2$ quarks

$$
\begin{aligned}
A^{\bar{Q}} & =\left[\begin{array}{ccc}
A_{1} & +B_{3} & -B_{2} \\
-B_{3} & A_{2} & +B_{1} \\
+B_{2} & -B_{1} & A_{3}
\end{array}\right], \\
B^{\bar{Q}} & =\left[\begin{array}{lll}
-B_{1} & -A_{3} & +A_{2} \\
+A_{3} & -B_{2} & -A_{1} \\
-A_{2} & +A_{1} & -B_{3}
\end{array}\right],
\end{aligned}
$$

and

$$
\begin{aligned}
P^{\bar{Q}} & =\left[\begin{array}{ccc}
p_{1}^{1} & +x_{3}^{1} & -x_{2}^{1} \\
-x_{3}^{2} & p_{2}^{2} & +x_{1}^{2} \\
+x_{2}^{3} & -x_{1}^{3} & p_{3}^{3}
\end{array}\right], \\
X^{\bar{Q}} & =\left[\begin{array}{lll}
-x_{1}^{1} & -p_{3}^{1} & +p_{2}^{1} \\
+p_{3}^{2} & -x_{2}^{2} & -p_{1}^{2} \\
-p_{2}^{3} & +p_{1}^{3} & -x_{3}^{3}
\end{array}\right] .
\end{aligned}
$$

(2) for the $\bar{I}_{3}=+1 / 2$ antiparticles of the $I_{3}=-1 / 2$ quarks:

$$
\begin{aligned}
A^{\bar{Q}^{\prime}}= & {\left[\begin{array}{ccc}
A_{1} & +B_{3} & -B_{2} \\
-B_{3} & A_{2} & +B_{1} \\
+B_{2} & -B_{1} & A_{3}
\end{array}\right]=A^{\bar{Q},} } \\
B^{\bar{Q}^{\prime}}= & -\left[\begin{array}{lll}
-B_{1} & -A_{3} & +A_{2} \\
+A_{3} & -B_{2} & -A_{1} \\
-A_{2} & +A_{1} & -B_{3}
\end{array}\right]=-B^{\bar{Q}},
\end{aligned}
$$

and

$$
\begin{aligned}
P^{\bar{Q}^{\prime}}= & {\left[\begin{array}{ccc}
p_{1}^{1} & +x_{3}^{1} & -x_{2}^{1} \\
-x_{3}^{2} & p_{2}^{2} & +x_{1}^{2} \\
+x_{2}^{3} & -x_{1}^{3} & p_{3}^{3}
\end{array}\right]=P^{\bar{Q}}, } \\
X^{\bar{Q}^{\prime}}= & -\left[\begin{array}{lll}
-x_{1}^{1} & -p_{3}^{1} & +p_{2}^{1} \\
+p_{3}^{2} & -x_{2}^{2} & -p_{1}^{2} \\
-p_{2}^{3} & +p_{1}^{3} & -x_{3}^{3}
\end{array}\right]=-X^{\bar{Q}} .
\end{aligned}
$$

The pattern of relative signs between all of $(45,48,51,53)$ is closely connected to the structure of the eigenvalues of $I_{3}$ and $Y$. Had we started from the other representation of the algebraic counterparts of the $I_{3}=+1 / 2$ quarks, i.e. from $\left(A^{Q(\dagger)}, B^{Q(\dagger)}\right)$, the relevant phase space representations for all other quarks and antiquarks would have changed accordingly. In particular, this would amount to a change of sign in front of all physical position coordinates appearing in $P^{Q}, P^{Q^{\prime}}, P^{\bar{Q}}$, and $P^{\bar{Q}^{\prime}}$. The relative signs between the components in a given direction in phase space would have stayed unchanged, however. Thus, e.g. if $x_{k}^{n}$ 
enters into canonical momenta of a quark of colour $n \neq k$ with a given (positive or negative) sign, then $x_{k}^{m}$ (for $n \neq m \neq k$ ) enters with the opposite sign. Furthermore, the relative connection between quarks and antiquarks is independent of whether we start from $\left(A^{Q}, B^{Q}\right)$ or from $\left(A^{Q(\dagger)}, B^{Q(\dagger)}\right)$. Just as for leptons and antileptons, the quarks and antiquarks are connected by a reflection in (physical) position space $($ see $(45,51)$ or $(48,53))$, which may be symbolically written as:

$$
\begin{aligned}
& P^{\bar{Q}}\left(p_{k}^{n}, x_{l}^{m}\right)=P^{Q}\left(p_{k}^{n},-x_{l}^{m}\right), \\
& X^{\bar{Q}}\left(p_{k}^{n}, x_{l}^{m}\right)=X^{Q}\left(p_{k}^{n},-x_{l}^{m}\right),
\end{aligned}
$$

accompanied by the change of sign of the free-standing $i$. This, together with $(26,27)$ for leptons, is the phase-space counterpart of the standard interpretation of antiparticles as "particles moving backward in time'. Yet, in the phase-space-based interpretation the concept of 'time' is not introduced in any explicit way. This seems to fit well into the philosophy of timeless quantum gravity.

\section{Discussion and Outlook}

In the classical limit, when one goes with the Planck constant to zero, the $i$ ceases to contribute on the r.h.s. of position-momentum commutation relations. With the quantum $i$ absent, the difference between particles and antiparticles (of a given isospin)-when interpreted in terms of classical phase-space concepts-reduces to the reflection of position space alone. Note that this might have been expected on the basis of the appearance-already in the case of the classical 3D harmonic oscillator (i.e. no $i$ ) —of the $U(1) \otimes S U(3)$ symmetry group. There is nothing wrong with the existence of two different classical interpretations of the connection between particles and antiparticles: the Stückelberg-Feynman interpretation and the phase-space interpretation herein proposed simply constitute different faces of the same coin. The advantage of the phase-space picture is that it permits a timeless classical interpretation of the connection between particles and antiparticles, with timelessness being a feature deemed welcome for the development of quantum gravity $[6,8]$.

A very interesting feature of $(45,48)$ and their charge-conjugate versions of $(51,53)$ is the pattern of signs in front of the physical components of phase-space variables (e.g. $-x_{3}^{1}$ and $+x_{2}^{1}$ in (45)). The negative signs cannot be all simultaneously changed into positive ones by a redefinition of the type:

$$
\left(x_{3}^{1}, x_{2}^{1}\right) \rightarrow\left(x_{3}^{\prime \prime}, x_{2}^{\prime 1}\right) \equiv\left(-x_{3}^{1}, x_{2}^{1}\right)
$$

because the preservation of the physical content of (45) requires that such a redefinition be applied to all relevant position and momentum components simultaneously (i.e., in the case of the redefinition of (55), to $x_{3}^{n}, x_{2}^{n}$ with any $n$, and to the respective components of momenta). This pattern of signs follows from our physical assumptions (recall also the discussion just before Sect. 4.1).

Keeping in mind the pattern of signs in $(45,48,51,53)$, we now come to the issue of the additivity of quark momenta. As already stressed in the introduction, the physical momentum of a system of ordinary classical particles is obtained by adding the physical momenta of its components, independently of where (in the configuration space) the particles are located. This additivity carries over to the standard quantum formalism. In our approach 
there exist two seemingly 'natural', but different, ways in which the idea of the additivity of physical momenta of ordinary particles may be generalized to the quark sector. The two generalizations exist because the concepts of physical and canonical momenta coincide here for ordinary particles, but are not equivalent for quarks. One may therefore consider either the additivity of quark physical momenta, or the additivity of quark canonical momenta. The latter option is possible because - despite the theoretical edifices built - the additivity of physical momenta of (standardly defined) individual quarks has not been really tested, as these momenta are never measured. Indeed, we always measure the momenta of quark conglomerates (i.e. hadrons). In standard approaches we then assume that hadrons are built of quarks possessing properties of ordinary particles, and in particular, satisfying the standard way of ensuring the additivity of quark momenta. Now, the whole argument of bringing more symmetry between physical position and momentum variables suggests that it is the additivity of canonical momenta which is more natural within our scheme. Consequently, let us accept that the additivity of the (operators of) physical momenta of ordinary particles (and antiparticles) is a special case of the general principle of additivity of (the operators of) canonical momenta. Then, one can apply the concept of additivity of canonical momenta to the quark sector.

In order to gain some quasi-classical understanding of the situation, consider a quarkantiquark $q_{\underline{n}} \bar{q}_{\underline{n}}$ system for a fixed $n$ (i.e. no superpositions of $q \bar{q}$ pairs of different colour) and with a well-defined canonical momentum. Its description should then involve, in particular, ordinary addition of quark and antiquark physical momenta in the $n$-th direction. Due to the opposite signs with which quark and antiquark positions (for any combination of $I_{3}$ eigenvalues) enter into the expressions for the canonical momenta $\mathbf{p}^{Q n}, \mathbf{p}^{\bar{Q}^{n}}$, the addition of the appropriate components of the latter leads to the subtraction of the corresponding physical position coordinates in directions perpendicular to the physical momentum. Hence, while individual quarks possess translationally noninvariant canonical momenta, the translational invariance of their sum, relevant for the composite $q \bar{q}$ system, is ensured. This has some similarity to the idea of a translationally invariant string connecting quark and antiquark.

Another way of forming translationally invariant expressions from quark canonical momenta is through the addition of canonical momenta of three quarks of different colours. In this case-again thanks to the different signs with which the physical positions enter into the expressions for the canonical momenta-all three quarks will conspire together to form translationally invariant expressions, i.e. $x_{3}^{2}-x_{3}^{1}, x_{2}^{1}-x_{2}^{3}, x_{1}^{3}-x_{1}^{2}$ from (45). (The idea of quark conspiracy requires also that phase-space variables corresponding to quarks of different colours, when appropriately grouped, form ordinary vectors together.) On the other hand, a pair of quarks is not sufficient to form translationally invariant expressions. Clearly, all this looks just like the emergence of mesons and baryons as composite systems built from unobservable quarks.

Note that a classical picture requires talking about well-defined canonical positions in addition to well-defined canonical momenta. Here, however, in both meson and baryon cases we are not dealing with well-defined canonical positions as yet. Thus, in fact, we do not have strings 'stretching between' quarks: our quarks are still not fully localized in the ordinary 3D configuration space. The picture is still quantum.

In order to see if 'real' strings can be obtained, one would need a better understanding of the transition from the quantum to the classical description of Nature. This is the goal of all emergent-space programs including our 'emergent phase space' proposal, and falls obviously far beyond the scope of this paper. The quasi-classical limit of the quantum picture to which we are led is certainly weird. Yet, it follows in a logical way from fundamental 
assumptions which, in my opinion, look very natural. Consequently, I am inclined to believe that it captures an important aspect of physical reality.

The above discussion is intended to show that our proposal for the nature of quarks has a built-in capacity to explain the phenomenon of quark confinement within a stringlike description. Such a description does not have to be in conflict with the present fieldtheoretical QCD approach, which describes what happens at large momenta transfers, not at large position differences, and does it within a specific theoretical description framework, built upon the background of ordinary 3D space (or spacetime). In our approach, on the other hand, this classical background (together with the relevant gauge structure) is expected to emerge only later, and it is only then that more precise connections to the QCD description may be sought.

Additivity of ordinary physical momenta may be viewed as resulting from a limiting case of the additivity of angular momenta, when the point with respect to which angular momenta are evaluated is shifted to spatial infinity. Thus, additivity of physical momenta may be traced back to the quantum-level additivity of the spin operators of component particles. Analogously, additivity of the canonical momenta in the quark sector should also have a corresponding operator counterpart at the discrete quantum level. It is this counterpart that would presumably play an important rôle in the construction of properly behaving protohadronic composite systems.

\section{Summary}

In this paper we have proposed a phase-space interpretation of the connections between leptons, quarks, and their antiparticles. The interpretation is timeless-which might be relevant for quantum gravity - and amounts to different relations between physical and canonical phase-space variables. In particular, some components of the canonical momenta of quarks are identified with their physical positions, thus lacking invariance under translations. We suggested that the principle of the additivity of physical momenta for leptons (and other ordinary particles) is a special case of the additivity of canonical momenta in general. This generalization was then shown to lead to the emergence of translationally invariant expressions for the $q \bar{q}$ and $q q q$ systems, a mechanism conjectured to be intimately related to quark confinement and the existence of mesons and baryons.

Acknowledgement I would like to thank Enrico Prati for bringing the FQXi contest on the Nature of Time to my attention.

Open Access This article is distributed under the terms of the Creative Commons Attribution Noncommercial License which permits any noncommercial use, distribution, and reproduction in any medium, provided the original author(s) and source are credited.

\section{References}

1. Barbour, J.: Leibnizian time, Machian dynamics, and quantum gravity. In: Penrose, R., Isham, C.J. (eds.) Quantum Concepts in Space and Time, pp. 236-246. Oxford University Press, Oxford (1986)

2. Barbour, J.: Phys. Rev. D 47, 5422 (1993)

3. Barbour, J.: The nature of time. First prize of the FQXi The Nature of Time contest. arXiv:0903.3489 (2009)

4. Born, M.: Rev. Mod. Phys. 21, 463 (1949)

5. Harari, H.: Phys. Lett. B 86, 83 (1979) 
6. Kiefer, C.: Does time exist in quantum gravity? Second prize of the FQXi The Nature of Time contest. arXiv:0909.3767 (2009)

7. Pavšič, M.: The Lanscape of Theoretical Physics: A Global View, pp. 223-224. Kluwer, Dordrecht (2001). arXiv:gr-qc/0610061v2

8. Rovelli, C.: Phys. Rev. D 43, 442 (1991)

9. Shupe, M.A.: Phys. Lett. B 86, 87 (1979)

10. Wheeler, J.A.: From relativity to mutability. In: Mehra, J. (ed.) The Physicist's Conception of Nature, pp. 202-247. D. Reidel, Dordrecht (1973)

11. Zachos, C.K.: Int. J. Mod. Phys. A 17, 297 (2002)

12. Żenczykowski, P.: Acta Phys. Pol. B 38, 2053, 2631 (2007)

13. Żenczykowski, P.: Phys. Lett. B 660, 567 (2008)

14. Żenczykowski, P.: J. Phys. Conf. Ser. 174, 012032 (2009). arXiv:0901.2896

15. Żenczykowski, P.: J. Phys. A 42, 045204 (2009) 